\title{
Prática Mental, Terapia Ocupacional e Reabilitação no Acidente Vascular Cerebral
}

\author{
Mental Practice, Occupational Therapy, and \\ Rehabilitation in Stroke \\ Práctica Mental, Terapia Ocupacional y \\ Rehabilitación no Accidente Cerebrovascular
}

\author{
Thamyres Pereira Alves ${ }^{1}$, Gabriela Ferraz Jaime ${ }^{2}$, Adriene Damasceno \\ Seabra ${ }^{3}$, Glenda Miranda da Paixão4, Kátia Maki Omura5, Otavio \\ Augusto de Araujo Costa Folha6, Marcelo Marques Cardoso ${ }^{7}$
}

\begin{abstract}
1.Terapeuta ocupacional, Faculdade de Fisioterapia e Terapia Ocupacional, Universidade Federal do Pará. Belém-PA, Brasil. https://orcid.org/0000-0002-3380-7711

2.Terapeuta ocupacional, Faculdade de Fisioterapia e Terapia Ocupacional, Universidade Federal do Pará. Belém-PA, Brasil. https://orcid.org/0000-0003-1172-0698

3.Terapeuta ocupacional, Mestre, Docente Assistente da Faculdade de Fisioterapia e Terapia Ocupacional, Universidade Federal do Pará. Belém-PA, Brasil. https://orcid.org/0000-0002-4367-9098

4.Terapeuta ocupacional, Doutora, Docente Adjunta da Faculdade de Fisioterapia e Terapia Ocupacional, Universidade Federal do Pará. Belém-PA, Brasil. https://orcid.org/0000-0001-9479-2659

5.Terapeuta ocupacional, Doutora, Docente Adjunta da Faculdade de Fisioterapia e Terapia Ocupacional, Universidade Federal do Pará. Belém-PA, Brasil. https://orcid.org/0000-0001-5113-5317

6.Terapeuta ocupacional, Doutor, Docente Adjunto da Faculdade de Fisioterapia e Terapia Ocupacional, Universidade Federal do Pará. Belém-PA, Brasil. https://orcid.org/0000-0003-3694-9691

7.Terapeuta ocupacional, Doutor, Docente Adjunto da Faculdade de Fisioterapia e Terapia Ocupacional, Universidade Federal do Pará. Belém-PA, Brasil. https://orcid.org/0000-0002-4792-5110
\end{abstract}

\section{Resumo}

Introdução. Sobreviventes de Acidente Vascular Cerebral (AVC) podem ser reabilitados pela Terapia Ocupacional (TO) tradicional ou associada a procedimentos que carecem de exploração, como a Prática Mental (PM). Objetivo. Investigar a eficácia do treinamento de Atividades de Vida Diária (AVDs), mentalmente executadas, como complemento à reabilitação tradicional. Método. Em uma Instituição Federal de Ensino Superior, pacientes sobreviventes de AVC foram distribuídos em: controle (GC) e prática mental (GPM), admissão pelo Mini Exame do Estado Mental e teste baseado no Questionário Revisado de Movimento Imaginário (MIQ-RS). Avaliações e registros: Escala de Força Muscular (MRC), Estesiômetro e Medida de Independência Funcional (MIF). Tratamento: 20 sessões, uma hora de duração (50 minutos fundamentados em AVDs e 10 minutos de PM para o GPM ou de relaxamento para o GC). Resultados. Os testes físicos demonstram recuperação de Componentes dos Desempenho Ocupacional testados no GPM. A MIF atesta ganho funcional e sugere celeridade na reabilitação no GPM. Conclusão. a PM de AVDs, aliada ao programa de reabilitação tradicional, promoveu ganhos físico-funcionais.

Unitermos. Prática Mental; Reabilitação; Movimento; Acidente Vascular Cerebral; Terapia Ocupacional; Atividade de Vida Diária

\footnotetext{
Abstract

Introduction. Stroke Survivors can be rehabilitated by traditional Occupational Therapy (OT) or associated with procedures that need to be explored, such as Mental Practice (MP). Objective. To investigate the effectiveness of training in Activities of Daily Living (ADLs), mentally performed, as a complement to traditional occupational therapeutic intervention in rehabilitation. Method. In a Federal Institution of Higher Education, stroke survivors were grouped into: control (CG) and mental practice (GPM), admission by the Mini Mental State Examination (Mini Mental), and test based on the Revised Movement Imagery Questionnaire -
} 
Revised for Stroke (MIQ-RS). Assessment and recording: Muscle Strength Scale - Medical Research Council (MRC); Esthesiometer; and Functional Independence Measure (FIM). Treatment: 20 sessions, one hour duration (50 minutes based on ADLs and 10 minutes of MP or relaxation). Results. Physical tests demonstrate the recovery of Occupational Performance Components tested in the GPM. The FIM certifies the functional recovery and points out the speed on GPM rehabilitation. Conclusion. MP combined with the traditional ADL rehabilitation program, promotes physical-functional gains.

Keywords. Mental Practice; Rehabilitation; Movement; Stroke; Occupational therapy; Activities of Daily Living.

\section{Resumen}

Introducción. Los sobrevivientes de un Accidente Cerebrovascular (ACV) pueden ser rehabilitados por la Terapia Ocupacional (TO) tradicional o asociada con procedimientos que necesitan de exploración, como la Práctica Mental (PM). Objetivo. Investigar la efectividad de un entrenamiento de Actividades de la Vida Diária (AVDs), mentalmente ejecutadas, como complemento a la rehabilitación tradicional. Método. En una Institución Federal de Educación Superior, personas sobrevivientes de ACV se dividieron en: control (GC) y práctica mental (GPM), admisión por el Mini Examen del Estado Mental e prueba basada en el cuestionario revisado del movimiento imaginario (MIQ-RS). Evaluaciones y registros: Escala de Fuerza Muscular (MRC); Monofilamento de Semmes-Weinstein; y Medida de Independencia Funcional (MIF). Tratamiento: 20 sesiones, una hora de duración (50 minutos basados en AVDs e 10 minutos, PM para GPM o relajación para GC. Resultados. Las pruebas físicas mostraran la recuperación de los Componentes de Desempeño Ocupacional probados en el GPM. La MIF logra mejora funcional y sugiere una reabilitación de mayor velocidad en el GPM. Conclusión. La PM de las AVDs, asociada al programa de reabilitación tradicional, promueve ganancias físicas y funcionales.

Palabras clave. Práctica Mental; Reabilitación; Movimento; Accidente Cerebrovascular; Terapia Ocupacional; Actividades de la Vida Diária

Trabalho realizado no Ginásio Adulto da Faculdade de Fisioterapia e Terapia Ocupacional, Instituto de Ciências da Saúde, Universidade Federal do Pará (FFTO/ICS/UFPA), Belém-PA, Brasil.

Conflito de interesse: não

Recebido em: 05/07/2020

Aceito em: $18 / 12 / 2020$

Endereço para correspondência: Marcelo M Cardoso. Universidade Federal do Pará, Instituto de Ciências da Saúde, Faculdade de Fisioterapia e Terapia Ocupacional. R. Augusto Correa 01, Guamá. BelémPA, Brasil. CEP 66075-110. Fone Institucional: (91) 3201-8892. Cel Pessoal: (91) 9384-3048. E-mail: marcelomc@ufpa.br

\section{INTRODUÇÃO}

\section{O Acidente Vascular Cerebral (AVC) é a segunda maior} causa de morte no mundo e a terceira em instalação de disfunções neurológicas ${ }^{1}$. Os sobreviventes podem manifestar inúmeras sequelas que prejudicam a qualidade de vida, desde disfunções físicas a prejuízos $\operatorname{cognitivos}^{2}$ e, secundariamente, comprometimentos à independência e 
Atividades de Vida Diária (AVD) ${ }^{3}$. Dos sobreviventes, estimase que mais da metade não conseguem fazer uso funcional dos membros superiores ${ }^{4}$, consequência principalmente dos déficits de força muscular e acometimento da percepção sensitiva5. Estas e outras variáveis físicas podem ser conceituadas como Componentes de Desempenho6.

Frequentemente, as sequelas a Componentes de Desempenho são as mais trabalhadas na reabilitação conduzidas por terapeutas ocupacionais, que objetivam ganhos funcionais para alcançar a independência e a autonomia em ocupações ${ }^{7}$. Os atendimentos clássicos da Terapia Ocupacional (TO), para esta clientela, podem abranger desde a execução de tarefas simples do cotidiano ${ }^{8}$ ao treino de $\operatorname{AVDs}^{9}$ e, abordagens não específicas da profissão, como a Terapia por Contensão Induzida ${ }^{10}$. Além dos procedimentos tradicionais, estudos em reabilitação mediada pela TO experimentam métodos e técnicas inovadoras como a Prática Mental (PM) que, conceitualmente, trabalha com a idealização de uma ação motora planejada sem que haja o movimento de qualquer parte do corpo ${ }^{11}$. Na literatura, a PM coleciona resultados gerais promissores em pessoas com sequelas neurológicas agudas $^{12}$. Assim, surgem iniciativas com movimentos funcionais na PM como abordagem complementar à reabilitação.

Prova disso, é um estudo pioneiro conduzido por Page et al. ${ }^{13}$ que analisaram a eficácia da PM na recuperação funcional de pacientes com sequelas motoras após um AVC. 
Para tanto, usaram atividades de baixa complexidade cinemática e neurofuncional, como: alcançar e pegar um copo, folhear páginas de um livro e o uso de uma caneta para escrever frases curtas. Os resultados evidenciaram melhora motora no membro afetado, com ganho funcional, além de benefícios em habilidades gerais na execução de $\mathrm{AVDs}^{13}$. Procedimentos semelhantes foram repetidos desde então com resultados animadores ${ }^{14,15}$. Para fomentar estas iniciativas, o presente estudo tem como originalidade a abordagem de práticas mais complexas, ligadas ao treino de AVDs mentalmente orientadas e, posteriormente, fisicamente executadas para descrever seus efeitos na reabilitação de pessoas sobreviventes de AVC.

Assim, o aumento de complexidade da PM a partir do treinamento de AVDs, conduzidas mentalmente, pode ser uma opção complementar às sessões de reabilitação tradicional. Por esse contexto, objetivou-se averiguar a aplicabilidade e a eficácia de tarefas motoras simples, baseadas em AVDs, mentalmente executadas, de modo complementar ao plano de intervenção terapêutico ocupacional em reabilitação de pacientes pós AVC.

\section{MÉTODO}

\section{Amostra}

Estudo randomizado, aprovado por comitê de ética em pesquisa, parecer número $1.337 .714 / 2015$. Todos os procedimentos ocorreram na clínica escola de reabilitação de 
uma Instituição Federal de Ensino Superior (IFES). Critérios de inclusão: indivíduos maiores de 18 anos; que sofreram um AVC há menos de seis meses; com manifestações de sequelas neurológicas de cunho motor/sensorial com duração superior a 24 horas; com alta ambulatorial há pelo menos três meses; pontuação de corte definido no teste de rastreio cognitivo pela escolaridade do participante. Critério de exclusão: casos de AVC em estágio agudo, alterações cognitivas e/ou psicoafetivas causadas pelo AVC, falta de aderência ao serviço proposto e dificuldades para idealizar ambientes e/ou mentalizar movimentos sugeridos. Vinte e dois pacientes foram avaliados inicialmente para inserção ao programa de atendimentos (Figura 1).

\section{Procedimentos}

Partes dos procedimentos realizados foram adaptados de Cardoso et al. ${ }^{16}$. Resumidamente: na admissão, o filtro dos critérios de elegibilidade iniciou pela aplicação do Mini Exame do Estado Mental (MEEM) para rastreio cognitivo, cujo ponto de corte foi definido pela escolaridade. O MEEM foi aplicado para identificar participantes com boa compreensão de comandos e sem comprometimento cognitivo causado pelo AVC, que é uma função cerebral importante no momento de idealização, requisito necessário à PM aqui proposta. Os aprovados, seguiram para o teste baseado no Questionário Revisado de Movimento Imaginário (QRMI), uma tradução livre aqui utilizada do Movement Imagery Questionnaire - Revised for Stroke (MIQ-RS) ${ }^{17}$. 
Trata-se de um instrumento com 14 itens, sobre movimentos executados fisicamente sucedida à reprodução mentalmente. O participante pontua de 1 a 7 , identificando o quanto foi difícil idealizar o movimento (1 ponto), ao quanto foi muito fácil o idealizar ( 7 pontos) conforme descrito por Butler et al. ${ }^{17}$. O teste foi aplicado para selecionar os participantes com habilidade mínima para reprodução mental de práticas sugeridas. Aqui, foi definido o valor de corte em 70\% da pontuação máxima do QRMI, para admissão.

Figura 1. Fluxograma da pesquisa. Exposição resumida do conjunto de procedimentos entre os grupos da pesquisa desde a admissão à alta e encaminhamento dos pacientes.

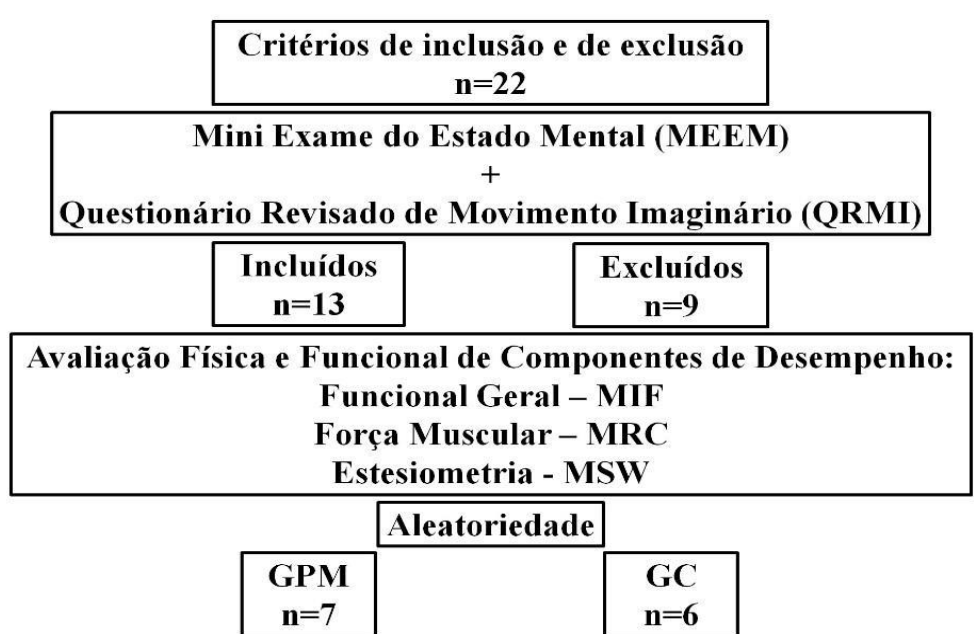

\begin{tabular}{|l|} 
Tempo de Tratamento: 10 semanas: \\
- 20 sessões \\
- 2 vezes por semana \\
- $1^{\text {a }}$ Sessão: Admissão \\
- 10 Sessão: Reavaliação \\
- 20 Sessão: alta \\
Duração da sessão: 60 minutos \\
- 50 minutos: Reabilitação Tradicional (AVD) \\
- 10 minutos: Prática Mental (AVD)
\end{tabular}

Tempo de Tratamento: 10 semanas:

- 20 sessões

2 vezes por semana

- ${ }^{\text {a }}$ Sessão: Admissão

- 10ªssão: Reavaliação

- 20ª Sessão: alta

Duração da sessão: 60 minutos

- 50 minutos: Reabilitação Tradicional (AVD)

- 10 minutos: Relaxamento Mental (Inerte)
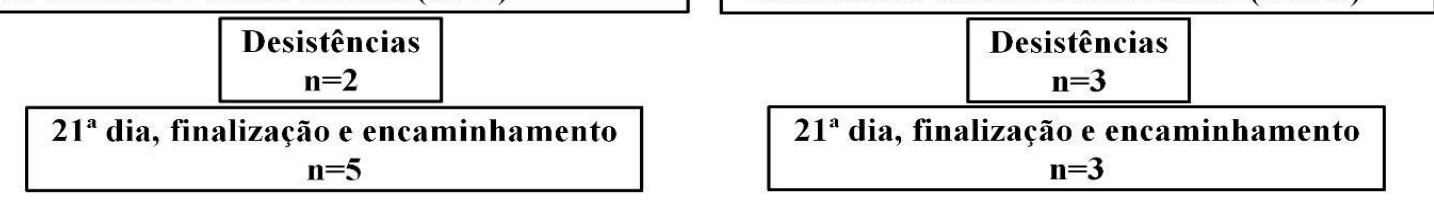
Os aprovados seguiram para o preenchimento de anamnese geral para coleta de dados pessoais e ocupacionais, focada em AVDs e atividades de interesse dos participantes. Posteriormente, estes foram aleatoriamente distribuídos entre os grupos da pesquisa.

Após o ordenamento entre os grupos, os participantes foram submetidos à avaliação física funcional centrada em Componente de Desempenho Ocupacional ${ }^{16}$, com os seguintes instrumentos: Medida de Independência Funcional (MIF), que busca averiguar o nível independência do sujeito no seu cotidiano; Escala de Força Muscular (Medical Research Council - MRC), o qual testa a força muscular nos membros inferiores e superiores; e Avaliação de sensibilidade pelo estesiômetro do tipo monofilamento de Semmes-Weinstein (MSW) que, aqui, acompanhou o perfil da percepção tátil dos membros superiores e inferiores.

\section{Tratamento}

Após a admissão, seguida pela avaliação e composição dos grupos de tratamento: Prática Mental (GPM) ou Controle (GC), foram executadas 20 sessões de atendimentos, duas vezes por semana, por dez semanas. Nesse tempo, foram realizados três momentos de avaliação: admissão (1 ${ }^{a}$ sessão), reavaliação ( $10^{a}$ sessão) e alta (20a sessão). Mais um dia foi acrescentado ( $21^{a}$ dia) para dar orientações e retorno aos participantes quanto a sua evolução ou dar encaminhamento para continuidade em outros serviços, caso 
o paciente ainda necessitasse de atendimento especializado (Figura 1).

Na primeira sessão, até quatro pesquisadores fizeram o primeiro registro de base das condições funcionais (MIF) e físicas do participante, avaliando os componentes de desempenho relacionados à força muscular (MRC) e à sensibilidade (MSW). Esses procedimentos de coleta de dados e de acompanhamento foram repetidos na $10^{a}$ e $20^{a}$ sessões.

Resumidamente, as sessões ocorreram da seguinte forma: cada sessão durou 60 minutos, sendo os primeiros 50 minutos de Terapia Ocupacional tradicional, focada em AVDs e, os últimos 10 minutos, foram empregados para idealizar mentalmente os movimentos de uma AVD préselecionada (GPM) ou realizar um relaxamento mental dirigido (GC). Na primeira parte da sessão, os participantes de ambos os grupos passaram por treinos comuns de AVDs, cuja base do procedimento foi repetida similarmente entre duas a três sessões consecutivas, dependendo do estado e da necessidade do paciente, tal como: preparação de refeições simples e alimentação, higienização das mãos, troca de peças de vestuário e autocuidados. Na segunda parte da sessão, apenas o GPM realizou as AVDs supracitadas mentalmente praticadas; e somente o GC foi orientado a idealizar cenários e sensações relaxantes inertes (Figura 1). Ambos sem envolver qualquer tipo de movimento. Roteiros preestabelecidos para cada grupo foram aplicados para garantir o rigor da sequência dos 
comandos e orientações, sendo repetidas de duas a três sessões consecutivas, dependendo da complexidade da prática mental eleita ou do cenário de relaxamento.

\section{Registro e Acompanhamento}

Os procedimentos de análises dos resultados e a estatística foram realizados após a tabulação dos dados gerados nos grupos. O GC serviu como parâmetro de comparação com os resultados do GPM e, assim, definir a evolução e o limiar de recuperação, tanto no intra como no extragrupo. Resumidamente: os dados obtidos pela MIF, MRC e MSW foram separados e organizados. Posteriormente, foram temporalmente agrupados, nos momentos de checagem, para as comparações pertinentes.

Dos três instrumentos de testes utilizados, foram extraídos os valores numéricos das escalas para a análise estatística comparativa entre os grupos e, a partir do valor da média apurada, enquadrou-se o grupo na condição qualitativa descritiva da respectiva escala eleita. Esta descrição também foi usada nas inferências dos resultados do grupo. Os dados da MIF e da MSW receberam tratamentos particulares semelhantes aos realizados por Cardoso et al. ${ }^{16}$. A seguir, os procedimentos com as três escalas são brevemente apresentados:

Medida de Independência Funcional. Para análise dos dados obtidos pela MIF, procedeu-se por entrevista direta. Ressalta-se que a MIF é uma escala de 18 itens, agrupadas 
em seis domínios. Cada item é pontuado em escala de 1 a 7, correspondendo, respectivamente, à dependência total e à independência completa, assim a pontuação total varia de 18 a 126 pontos.

Contudo, na admissão, os pacientes não manifestaram déficits em todos os 18 itens da MIF. Por isso, padronizou-se a exclusão do item que pontuasse o valor de independência completa (7 pontos), considerando-se apenas o registro dos itens com valores iguais ou menores que seis pontos, sem os somar ao final. Assim, nos três tempos de avaliação, extraiuse a média e o desvio padrão apenas dos itens com algum nível de dependência para gerar a análise evolutiva entre os grupos.

Estesiometria. Trata-se de um instrumento analógico, constituído por um conjunto de seis monofilamentos de espessuras variáveis (Sorri-Bauru ${ }^{\circledR}$ ), associados a cores, onde o mais delicado é o verde e o mais espesso, o rosa, (Quadro 1). Relacionou-se à cor preta a ausência de resposta por falta de percepção tátil do paciente.

Os dados estesiométricos passaram por artifício matemático sustentado pela ampla dispersão da secção transversal de cada monofilamento por unidade de área, expresso em grama-Força (gF). Por isso, optou-se pela conversão numérica em valores de logaritmo de base 10 (log10), na qual a dispersão se tornou menos discrepante numericamente ${ }^{18}$. 
Quadro 1. Conversão do valor nominal de cada monofilamento do estesiômetro para $\log 10^{18}$.

\begin{tabular}{|l|c|c|c|}
\hline COR & Gf & $\mathbf{x 1 0 0}$ & LOG 10 \\
\hline VERDE & 0,05 & 5 & 0,6990 \\
\hline AZUL & 0,2 & 20 & 1,3010 \\
\hline VIOLETA & 2,0 & 200 & 2,3010 \\
\hline VERMELHO & 4,0 & 400 & 2,6021 \\
\hline LARANJA & 10,0 & 1000 & 3,0000 \\
\hline ROSA & 300,0 & 30000 & 4,4771 \\
\hline PRETO & 900,0 & 90000 & 4,9542 \\
\hline
\end{tabular}

Resumidamente, para se evitar valores numéricos negativos no log10, cada valor nominal das cores dos monofilamentos foram multiplicados por $100 \mathrm{e}$, na sequência, transformados em logaritmos de base 10 (Quadro $1)^{18}$. Posteriormente, os valores obtidos foram agrupados nos três tempos de avaliação para extração da média e do desvio-padrão usados na comparação entre os grupos.

Força Muscular. Foi usada a Escala de Pesquisa Médica de Council, trata-se de um teste qualitativo do grau de força muscular com notação numérica de 0 a 5 pontos, no qual o grau zero representa a paralisia completa e o grau cinco, força muscular normal. Todas as mensurações foram realizadas pelo mesmo avaliador nos três tempos de registro. As médias do grau de força muscular dos membros, tanto do lado ipsilesional quanto contralesional, foram avaliados para gerar inferência evolutiva em resposta ao tratamento. Resumidamente: nos três tempos de checagem, o mesmo avaliador qualificou a força muscular nos membros 
superiores: ombro (abdução), cotovelo (flexão/ extensão) e punho (flexão/extensão). Os pontos testados foram agrupados para gerar a média e o desvio-padrão.

\section{Análise Estatística}

Os dados obtidos foram tabulados e agrupados para gerar comparações nos três tempos de avaliação do estudo. Os parâmetros de análise foram os lados ipsi e contralesional nos grupos GC e GPM. Por isso, aplicou-se a Estatística Descritiva pelo uso da Análise de Variância (ANOVA), dois critérios, em todas as análises, e Pós-teste de Fisher com índice de significância delimitada para valores de $p<0,05$. Os gráficos foram plotados em colunas para expressar as médias \pm desvio padrão. Programa GraphPad, Prism - 6.0.

\section{RESULTADOS}

\section{Prática mental favorece o ganho de força muscular}

Descritivamente, o resultado de força muscular (Figura 2) possibilita a inferência que, após o AVC, o hemicorpo ipsilesional apresenta sutil acometimento motor, comparado ao evidente prejuízo no hemicorpo contralesional. $\mathrm{Na}$ admissão, o estado físico entre os grupos aponta igual déficit na força muscular entre GPM e GC. Na alta, os grupos ganharam força muscular a valores equivalentes ao patamar de normalidade. 
Figura 2. Força Muscular. Grupos: Controle (hemicorpos: ipsilesional e contralesional) e prática mental (hemicorpos: ipsilesional e contralesional). Coleta de dados: Admissão (1 ${ }^{a}$ Sessão), avaliação (10a sessão) e alta (20a sessão). Asterisco $(p<0,05)$, comparação no mesmo hemicorpo. Hashtag $(p<0,05)$, comparação entre hemicorpos. Signum sectiōnis $(p<0,05)$, comparação entre grupos.

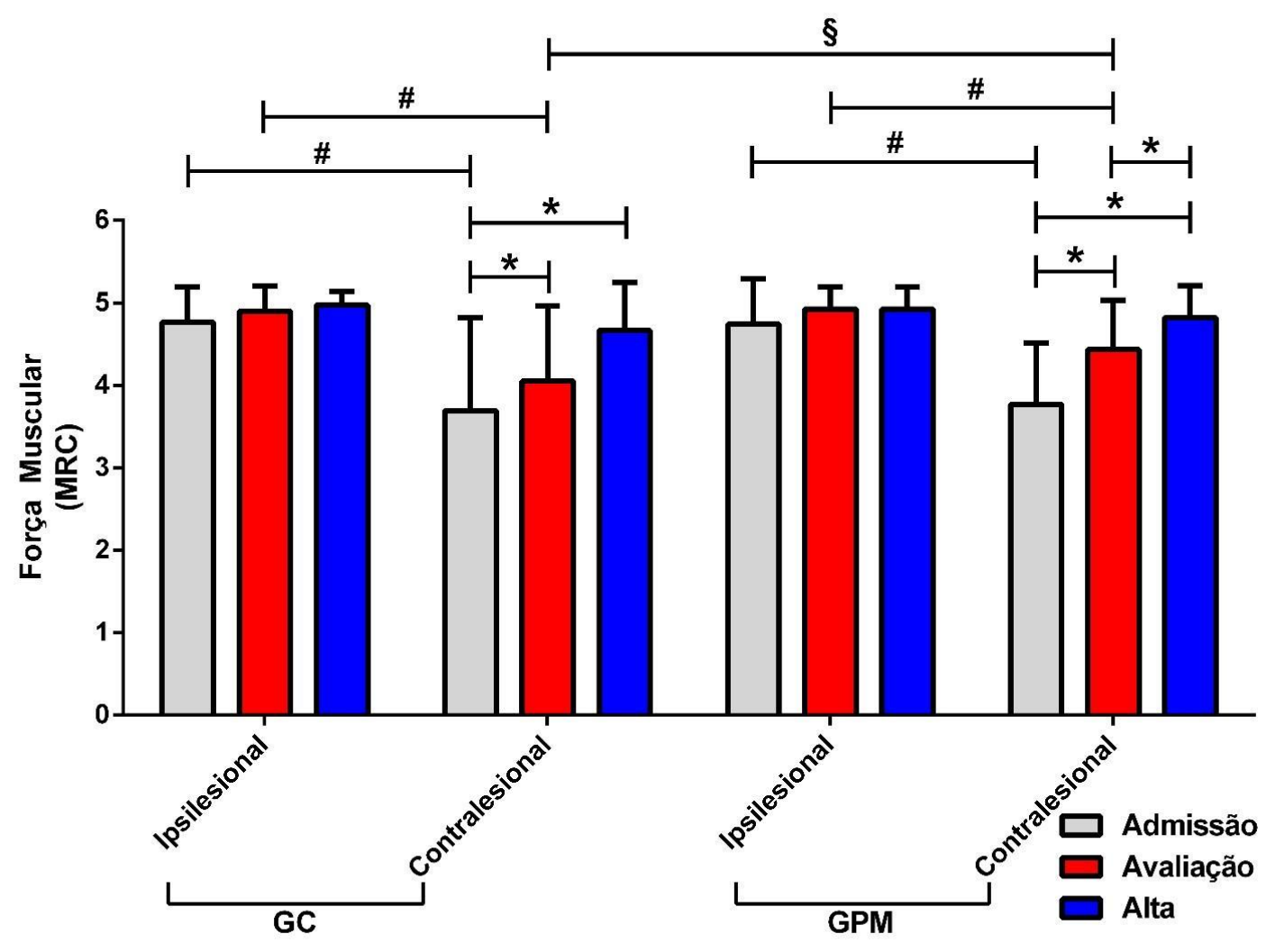

De outra forma, no GC é perceptível a evolução entre a

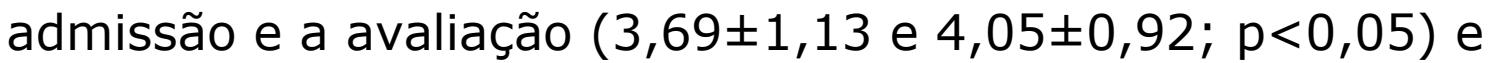

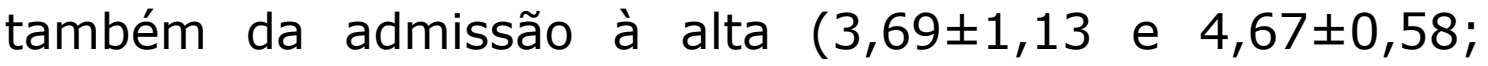
$p<0,05)$. Evolução similar ao GPM, tanto entre a admissão e a avaliação $(3,77 \pm 0,74$ e $4,44 \pm 0,60 ; p<0,05)$, quanto entre a admissão e a alta $(3,77 \pm 0,74$ e 4,82 $\pm 0,39 ; p<0,05$; Figura 2). Entre os grupos, a evolução da $10^{a}$ sessão, enfatiza-se que o GPM apresenta um salto no ganho de força muscular, comparado ao resultado médio do GC. A partir do qual, os 
grupos manifestaram comportamento evolutivo semelhante até a alta. No GPM, destaca-se a evolução entre a avaliação (10a sessão) e a alta (20a sessão), que não acontece no GC (Figura 2).

Desta forma, a descrição para esse Componente de Desempenho sugere que os grupos recuperam a força muscular de forma semelhante (Figura 2). Pelo exposto, superficialmente, pode-se inferir que o ganho de força muscular é beneficiado pela Terapia Ocupacional, com ou sem a prática mental. Contudo, entre os grupos, tanto a diferença estatística na avaliação da $10^{a}$ sessão, quanto a significância estatística de recuperação, entre a $10^{\mathrm{a}}$ e a $20^{\mathrm{a}}$ sessões, apenas no GPM, faz acreditar que a PM, baseada em AVDs, tem tendência de recuperação da força muscular.

\section{A prática mental melhora a resposta da percepção sensitiva}

Os resultados da estesiometria consolidam os benefícios da PM, pela idealização de AVDs na recuperação de Componentes de Desempenho. Descritivamente, como também observado na força muscular, aqui, nos dois grupos, o hemicorpo ipsilesional expressa algum grau de déficit de percepção sensitiva (Figura 3 ). Já o hemicorpo contralesional, como esperado, é o que manifesta maior prejuízo. Ao final, o hemicorpo contralesional do GPM tem melhor resultado que aquele observado no GC. A contundência da resposta no GPM o faz se igualar à normalidade da percepção tátil. Resposta não observada no GC. 
Figura 3. Estesiometria. Dados apresentados em Log10 correspondendo aos estratos das cores dos monofilamentos. Grupos: Controle (hemicorpos: ipsilesional e contralesional) e prática mental (hemicorpos: ipsilesional e contralesional). Coleta de dados: Admissão ( $1^{\text {a }}$ Sessão), avaliação (10a sessão) e alta (20a sessão). Asterisco $(p<0,05)$, comparação no mesmo hemicorpo. Hashtag $(p<0,05)$, comparação entre hemicorpos.

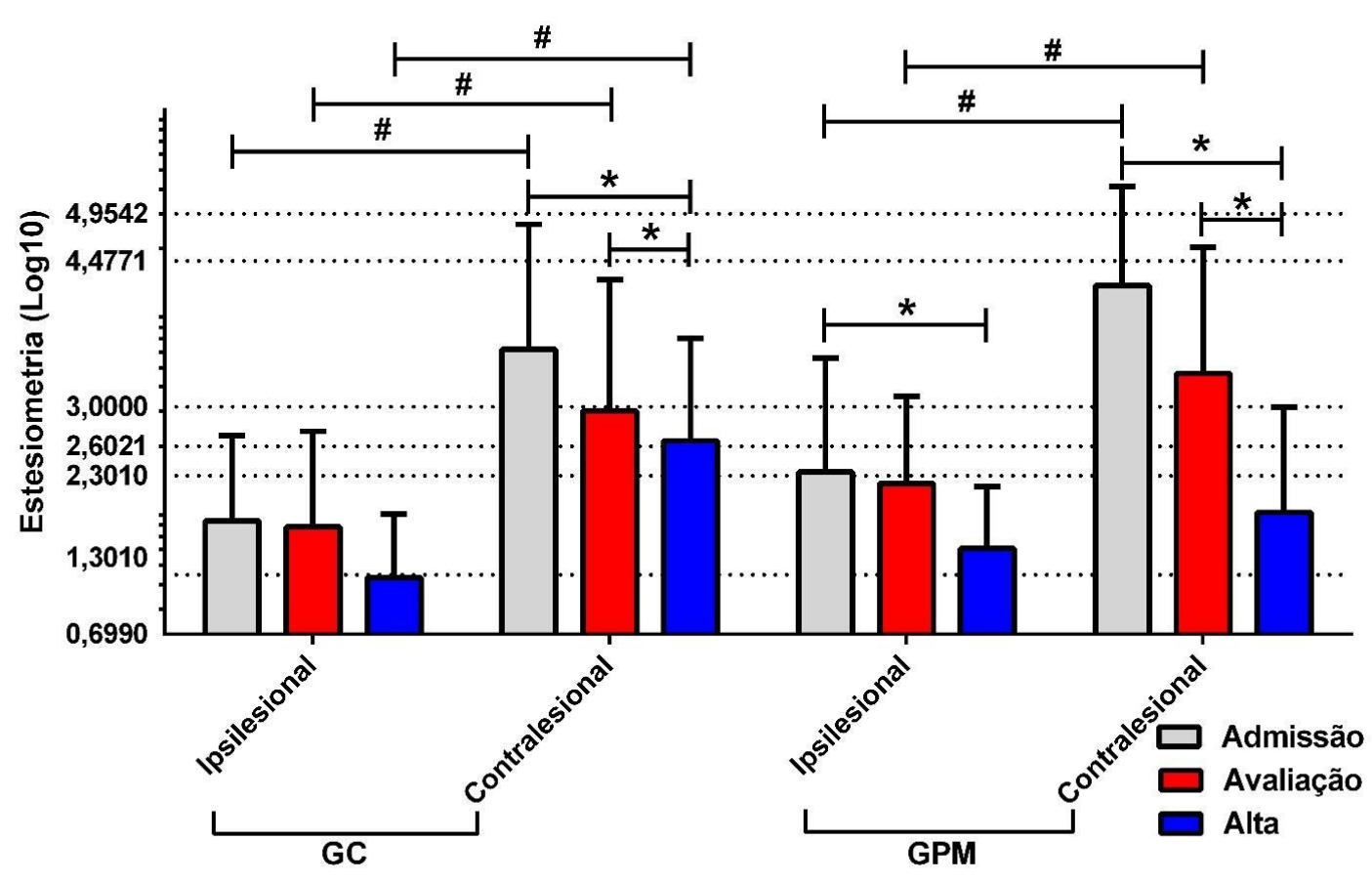

Qualitativamente, pelo padrão de cores dos monofilamentos do estesiômetro, o hemicorpo contralesional do GC, na admissão, manifesta percepção sensitiva compatível com a cor laranja (Figura 3). Também na $1^{\text {a }}$ sessão, a mesma cor representa a situação média no GPM. $\mathrm{Na}$ alta, o GC passa a ser representado pelo monofilamento vermelho, ao passo que o GPM chega a ser representado pelo monofilamento azul. Esses resultados sugerem maior ganho de precisão na percepção sensitiva no GPM, quando comparado ao GC. 
No hemicorpo contralesional do GC não há mudança da

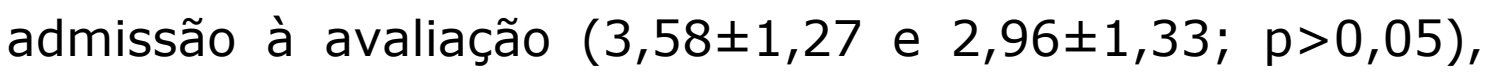
mas há entre a avaliação e a alta $(2,96 \pm 1,33$ e 2,65 1,04; $p<0,05)$. Mesmo apresentando resposta positiva ao tratamento, na alta, a percepção tátil no GC não se aproxima da faixa de normalidade observado no hemicorpo ipsilesional, pois entre os lados há diferença $(1,27 \pm 0,65$ e $2,65 \pm 1,04 ; p<0,05)$, evidenciando ausência de recuperação desse Componente de Desempenho no grupo.

No GPM, o lado contralesional tem comportamento evolutivo semelhante ao GC entre a admissão e a avaliação $(4,23 \pm 1,00$ e 3,34 1,$27 ; p>0,05)$ e da avaliação à alta

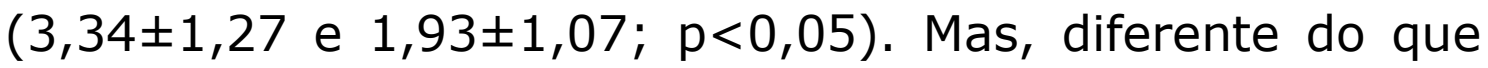
aconteceu no GC, o lado contralesional do GPM se igualou, ao hemicorpo ipsilesional no período da alta $(1,57 \pm, 62$ e $1,93 \pm 1,07, p>0,05)$. Esse resultado consolida a recuperação da percepção sensitiva no GPM a níveis de normalidade.

Desta forma, a análise do resultado qualitativo relacionada ao padrão de cores do estesiômetro é apresentada quanto a percepção da sensibilidade tátil, evidenciando que os dois grupos evoluem satisfatoriamente, mas é o GPM que, na alta, apresenta resultados que alcançam a faixa de normalidade (Figura 3). Assim, os achados da estesiometria acompanham a recuperação da força muscular (Figura 2), isso sugere que a prática complementar de idealização de AVDs tem potencial de induzir a recuperação de Componentes de Desempenho, durante a reabilitação mediada pela Terapia Ocupacional. 


\section{A MIF evidencia recuperação funcional precoce pela prática mental de AVDs}

A partir da descrição do resultado da MIF, percebe-se que os grupos iniciaram a reabilitação apresentando déficits funcionais proporcionalmente iguais. Contudo, o GPM evidenciou melhora precoce, ainda na avaliação da $10^{a}$ sessão (Figura 4). Esse achado reforça as inferências apresentadas sobre a força muscular (Figura 2) e a percepção tátil (Figura 3), como os Componentes de Desempenho aqui acompanhados.

No GC, a evolução foi sutil entre a admissão e a avaliação $(p>0,05)$. Por outro lado, entre a admissão e a alta

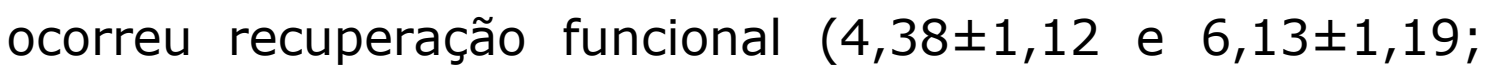
p<0,05; Figura 4). Já o GPM manifestou resultado mais proeminente. Entre a admissão e a avaliação, esse grupo evidenciou recuperação funcional $(4,13 \pm 0,83$ e 6,14 0,79; $p<0,05)$. Esse achado evoluiu numericamente da avaliação à alta, com sutil melhora, para consolidar a recuperação funcional $(p>0,05)$. Na avaliação de alta, o resultado funcional do GC demonstra redução no grau de dependência, como alcançado pelo GPM.

Figura 4. Medida de independência Funcional. Grupos: controle e prática mental. Coleta de dados: Admissão (1 a Sessão), avaliação (10a sessão) e alta (20a sessão). Asterisco $(p<0,05)$, comparação no mesmo grupo. Hashtag $(p<0,05)$, comparação entre grupos. 


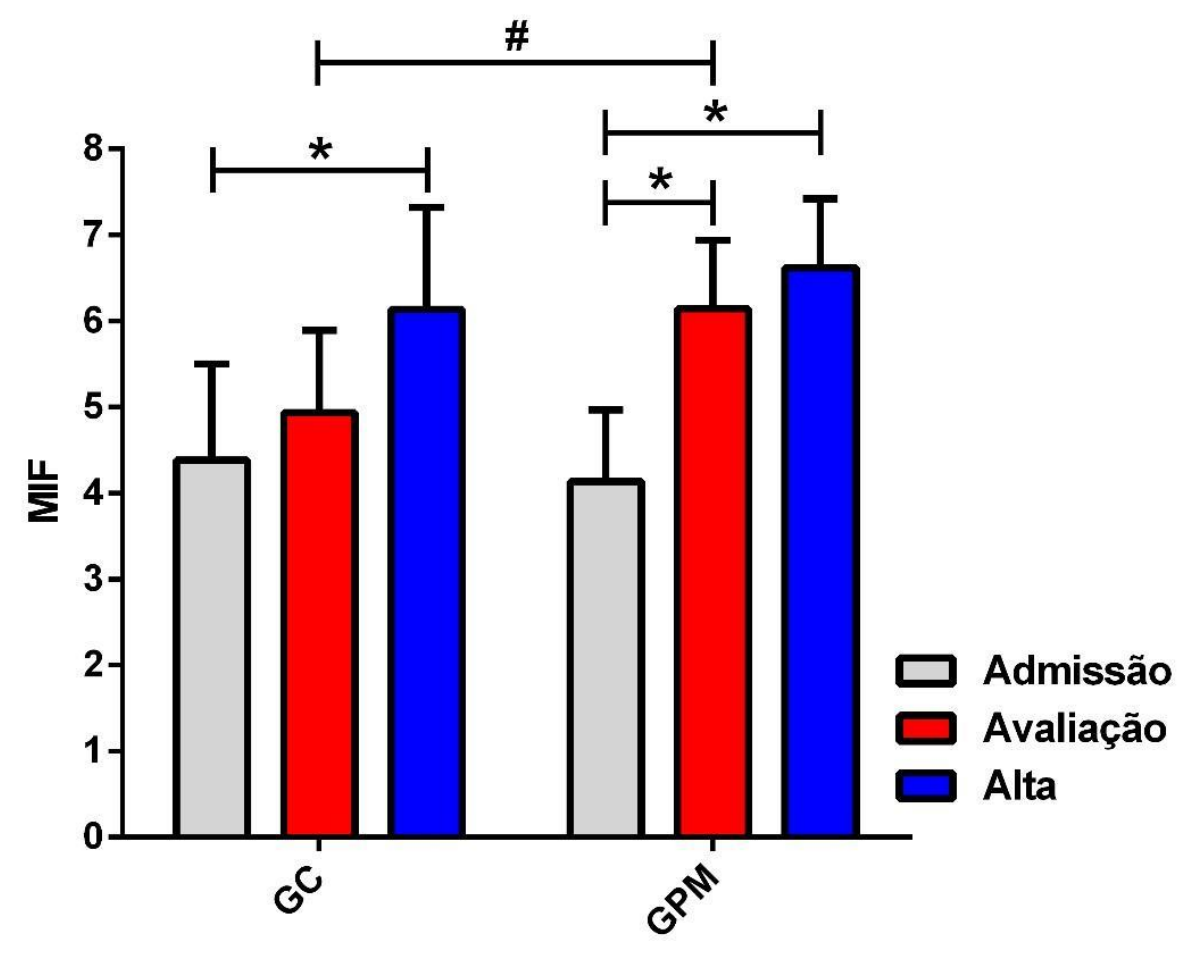

Esse achado sustenta os resultados relativos aos componentes de desempenho encontrados nesse estudo e sugere relevância na aplicação de PM, fundamentada em práticas específicas da Terapia Ocupacional, como aquelas inerentes ao treino de AVDs.

\section{DISCUSSÃO}

Tanto os Componentes de Desempenho quanto 0 Funcional sugerem que a prática terapêutica ocupacional, baseada no treino de AVDs, aplicada na reabilitação de pacientes sobreviventes de AVC, promoveu a reabilitação funcional em até 20 sessões (alta). Complementarmente, os achados aqui expostos trazem evidências que a PM, fundamentada em atribuições privativas da Terapia 
Ocupacional, pelo treino de AVDs, manifesta potencial para promover a reabilitação precoce. Esta reabilitação funcional foi sustentada pelos achados dos testes físicos aqui pesquisados. Diante disso, o principal achado do estudo sugere que a resposta ao tratamento do GPM é temporalmente mais eficiente que no GC. Assim, esses resultados demonstram benefícios potenciais da reabilitação neurológica com práticas específicas da Terapia Ocupacional e corrobora com a literatura especializada da área ${ }^{19,20}$, com a originalidade do emprego do treino de AVDs, mentalmente executadas.

A reabilitação imprime benefícios à variáveis do desempenho ocupacional na reabilitação de sequelas neurológicas ${ }^{21,22}$, como as relatadas no presente trabalho, que destaca resultados positivos com uso de procedimentos específicos da TO. Contraditoriamente, mesmo os resultados aparecendo nesta área, as evidências ainda são baixas ${ }^{9}$. Nesse sentido, os resultados aqui apresentados contribuem com acervo especializado de conhecimento da profissão e tendem a minimizar a carência de resultados relativos à intervenção na reabilitação com sobreviventes de AVC, baseada em treinos de AVDs.

Apesar das publicações especializadas emergentes e dos avanços da TO no campo da neurorreabilitação, dúvidas relativas a procedimentos ainda são persistentes, e a baixa reprodutividade deles faz falta no momento de comparar resultados e práticas inerentes da profissão, tal qual, a quantidade de intervenções e a frequência semanal das 
sessões de reabilitação ${ }^{23}$. No presente estudo, foram planejadas 20 sessões, de 1 de duração cada, duas vezes por semana, ao longo de dois meses e meio (10 semanas). Há dificuldade em padronizar estas variáveis entre as pesquisas para qualificar os resultados aqui encontrados. Por exemplo: foram utilizadas 4 intervenções com 1 hora de duração cada24; em outro estudo foi utilizada 30 sessões, uma hora cada, por um mês ${ }^{19}$; e foi executado em seis semanas, três vezes por semana, por uma hora cada sessão7 ${ }^{7}$ Nas pesquisas citadas, há evidências de algum grau de sucesso, assim os resultados aqui apresentados corroboram com a literatura. Do exposto, pode-se inferir que, práticas específicas da Terapia Ocupacional têm efeitos benéficos sobre a restauração de funções prejudicadas pelo AVC.

Os achados aqui apresentados, portanto, sugerem que a sistematização de práticas específicas da profissão, como o treino de AVDs, tem potencial para promover a reabilitação de pacientes sobreviventes de AVC independente do plus inserido no programa de reabilitação, evidenciado pela melhora em componentes de Desempenho Ocupacional e função demonstrados aqui. Assim, esses resultados corroboram com estudos anteriores ${ }^{6,25}$.

Reconhecidamente, indivíduos sobreviventes de AVC manifestam alterações significativas em sua capacidade funcional ${ }^{26}$. Tais déficits funcionais também foram encontrados nos dois grupos aqui ordenados. A escolha da MIF como parâmetro tanto de avaliação dos pacientes 
quanto na análise comparativa da resposta ao tratamento, nos momentos de checagem, foi bem-sucedida aqui, como em outros estudos ${ }^{27}$. Esta recuperação funcional, associada à PM, também foi encontrada com pessoas com sequelas neurológicas ${ }^{12}$. Foi possível verificar, também, que houve ganho importante em todos os períodos, sendo pelo grupo PM que obteve melhora acentuada, reforçando o achado de que a MIF é um instrumento útil e sensível para o acompanhamento dos sujeitos em reabilitação ${ }^{27}$.

Como abordado até aqui, e na literatura, procedimentos terapêuticos ocupacionais conduzem à restauração de habilidades prejudicadas pelo AVC $^{16}$. Por outro lado, de forma complementar, a PM proposta promoveu a reabilitação funcional com celeridade no GPM comparado ao GC. Classicamente, a PM é reconhecida por promover 0 reaprendizado de tarefas antes vivenciadas ${ }^{28}$. Acredita-se que esse efeito da PM tenha colaborado com a maior brevidade ao tratamento. Por se tratar de repetições mentais de atividades antes experienciadas, tanto de modo precedente ao AVC, como aqui treinadas fisicamente em cada sessão. Isso pode ter reforçado conexões neurais ${ }^{29}$ e ter favorecido o retorno de funções, benefício do treinamento de tarefas-específicas ${ }^{16}$.

Sobre o aspecto de procedimentos executados, com exclusões de itens da MIF, para o melhor aproveitamento de dados e de interpretação, há precedentes da literatura. Domínios da MIF foram trabalhados para gerar dados proporcionais, normalizados em forma de fração de 0-1, 
para discriminar o peso que os domínios motor e cognitivo tinham sobre resultados funcionais na reabilitação após o $A_{V} C^{30}$. Por isso, acredita-se que a exclusão de itens da MIF, aqui realizados, com patamar de normalidade na admissão, favoreceu a comparação evolutiva dos pacientes, por possibilitar a visualização das respostas, exclusivamente, dos itens com déficits registrados na $1^{\text {a }}$ sessão e, posteriormente, acompanhados.

\section{CONCLUSÃO}

Constatou-se, por fim, que a PM, aliada ao programa de reabilitação tradicional de Terapia Ocupacional é uma prática promissora que pode ser moldada às competências específicas da profissão por demonstrar no presente estudo potencial benefício físico e funcional, até de forma célere, representando independência modificada pela MIF aos pacientes. Esse estudo reforça a necessidade de investigações que refinem essa ferramenta inovadora incorporada à Terapia Ocupacional ao mesmo tempo que são demonstrados $\mathrm{O}$ benefício complementar da PM na reabilitação de pacientes sobreviventes de AVC em função do treino de AVDs. Estudos futuros podem instruir o uso de PM baseada em Atividades Instrumentais de Vida Diária (AIVDs) para gerar comparações destas com as AVDs e prosseguir no aprofundamento de novas pesquisas sobre a temática. 


\section{AGRADECIMENTOS}

Os autores agradecem o apoio da Pró-Reitoria de Pesquisa e Pós-Graduação (UFPA) e a colaboração dos terapeutas ocupacionais: Matheus de Almeida Paiva, Michelle Jacob da Cruz e Denis Carvalho Lobo.

\section{REFERÊNCIAS}

1.Wajngarten M, Silva GS. Hypertension and Stroke: Update on Treatment. Eur Cardiol 2019;14:111-5.

https://doi.org/10.15420/ecr.2019.11.1

2.Broussy S, Saillour-Glenisson F, García-Lorenzo B, Rouanet F, Lesaine $E$, Maugeais $M$, et al. Sequelae and Quality of Life in Patients Living at Home 1 Year After a Stroke Managed in Stroke Units. Front Neurol 2019;10:907. https://doi.org/10.3389/fneur.2019.00907

3. Warner G, Packer T, Villeneuve M, Audulv A, Versnel J. A systematic review of the effectiveness of stroke self-management programs for improving function and participation outcomes: self-management programs for stroke survivors. Disabil Rehabil 2015;37:2141-63. https://doi.org/10.3109/09638288.2014.996674

4.Rajagopalan V, Natarajan M, Gorthi SP, Padickaparambil S, Solomon JM. Effectiveness of a multifactorial context-enhancing functional therapy to promote functional arm use and recovery of stroke survivors: study protocol for a clinical trial. BMJ Open 2019;9:e023963. https://doi.org/10.1136/bmjopen-2018-023963

5.Sketch SM, Simpson CS, Crevecoeur F, Okamura AM. Simulating the impact of sensorimotor deficits on reaching performance. IEEE Int Conf Rehabil Robot 2017;2017:31-7.

https://doi.org/10.1109/ICORR.2017.8009217

6.Ahn SN, Yoo EY, Jung MY, Park HY, Lee JY, Choi YI. Comparison of Cognitive Orientation to daily Occupational Performance and conventional occupational therapy on occupational performance in individuals with stroke: A randomized controlled trial. NeuroRehabilitation 2017;40:285-92. https://doi.org/10.3233/NRE161416

7.Almhdawi KA, Mathiowetz VG, White M, delMas RC. Efficacy of Occupational Therapy Task-oriented Approach in Upper Extremity Poststroke Rehabilitation. Occup Ther Int 2016;23:444-56. https://doi.org/10.1002/oti.1447

8. Nott MT, Barden HLH, Chapparo C, Ranka JL. Evidence based practice and knowledge translation: A survey of Australian occupational therapy practice with clients experiencing neurocognitive impairments. Aust Occup Ther J 2020;67:74-82. https://doi.org/10.1111/1440$\underline{1630.12625}$ 
9.Legg LA, Lewis SR, Schofield-Robinson OJ, Drummond A, Langhorne P. Occupational therapy for adults with problems in activities of daily living after stroke. Cochrane Database Syst Rev 2017;7:CD003585. https://doi.org/10.1002/14651858.CD003585

10.Christie LJ, McCluskey A, Lovarini M. Constraint-induced movement therapy for upper limb recovery in adult neurorehabilitation: An international survey of current knowledge and experience. Aust Occup Ther J 2019;66:401-12. https://doi.org/10.1111/1440-1630.12567 11.Page SJ, Levine P, Sisto SA, Johnston MV. Mental practice combined with physical practice for upper-limb motor deficit in subacute stroke. Phys Ther 2001;81:1455-62. https://doi.org/10.1093/ptj/81.8.1455 12. Nam JS1, Yi TI, Moon HI. Effects of adjuvant mental practice using inverse video of the unaffected upper limb in subacute stroke: a pilot randomized controlled study. Int J Rehabil Res 2019;42:337-43. https://doi.org/10.1097/MRR.0000000000000368

13.Page SJ, Levine P, Leonard AC. Effects of mental practice on affected limb use and function in chronic stroke. Arch Phys Med Rehabil 2005;86:399-402. https://doi.org/0.1016/j.apmr.2004.10.002

14.Peters HT, Page SJ. Integrating Mental Practice with Task-specific Training and Behavioral Supports in Poststroke Rehabilitation: Evidence, Components, and Augmentative Opportunities. Phys Med Rehabil Clin N Am 2015;26:715-27.

https://doi.org/10.1016/i.pmr.2015.06.004

15. Kho AY, Liu KP, Chung RC. Meta-analysis on the effect of mental imagery on motor recovery of the hemiplegic upper extremity function. Aust Occup Ther J 2014;61:38-48. https://doi.org/10.1111/14401630.12084

16.Cardoso MM, Lobo DC, Cruz MJ, Monteiro RPA, Seabra AD, Paixão GM, et al. Abordagens específicas em Terapia Ocupacional em reabilitação após Acidente Vascular Encefálico. Rev Inter Bras Ter Ocup 2019;3:191-209. https://doi.org/10.47222/2526-3544.rbto21972

17. Butler AJ, Cazeaux J, Fidler A, Jansen J, Lefkove N, Gregg M, et al. The Movement Imagery Questionnaire-Revised, Second Edition (MIQRS) Is a Reliable and Valid Tool for Evaluating Motor Imagery in Stroke Populations. Evid Based Complement Alternat Med 2012;2012:497289. https://doi.org/10.1155/2012/497289

18. Azevedo PS, Cavalcante SM, Alves TP, Jaime GF, Nascimento AQ, Cardoso MM. A notação logarítmica como opção de registro objetivo da estesiometria: um estudo piloto. Conscientiae Saúde 2020;19:e17642. https://doi.org/10.5585/conssaude.v19n1.17642

19. Rowe VT, Neville M. Task Oriented Training and Evaluation at Home. Thorofare N J 2018;38:46-55.

https://doi.org/10.1177/1539449217727120

20.Lee MJ, Lee JH, Koo HM, Lee SM. Effectiveness of Bilateral Arm Training for Improving Extremity Function and Activities of Daily Living Performance in Hemiplegic Patients. J Stroke Cerebrovasc Dis 2017;26:1020-5.

https://doi.org/10.1016/j.jstrokecerebrovasdis.2016.12.008 
21. Hsieh YW, Chang KC, Hung JW, Wu CY, Fu MH, Chen CC. Effects of Home-Based Versus Clinic-Based Rehabilitation Combining Mirror Therapy and Task-Specific Training for Patients With Stroke: A Randomized Crossover Trial. Arch Phys Med Rehabil 2018;99:2399407. https://doi.org/10.1016/j.apmr.2018.03.017

22.Saeidi Borujeni M, Hosseini SA, Akbarfahimi N, Ebrahimi E. Cognitive orientation to daily occupational performance approach in adults with neurological conditions: A scoping review. Med J Islam Repub Iran 2019;33:99. https://doi.org/10.34171/mjiri.33.99

23.Serrada I, McDonnell MN, Hillier SL. What is current practice for upper limb rehabilitation in the acute hospital setting following stroke? A systematic review. NeuroRehabilitation 2016;39:431-8. https://doi.org/10.3233/NRE-161374

24.Adams RJ, Lichter MD, Krepkovich ET, Ellington A, White $M$, Diamond PT. Assessing upper extremity motor function in practice of virtual activities of daily living. IEEE Trans Neural Syst Rehabil Eng 2015;23:287-96. https://doi.org/10.1109/TNSRE.2014.2360149

25.Adams RJ, Lichter MD, Ellington A, White M, Armstead K, Patrie JT, et al. Virtual Activities of Daily Living for Recovery of Upper Extremity Motor Function. IEEE Trans Neural Syst Rehabil Eng 2018;26:252-60. https://doi.org/10.1109/TNSRE.2017.2771272

26.Galeoto G, Iori F, De Santis R, Santilli V, Mollica R, Marquez MA, et al. The outcome measures for loss of functionality in the activities of daily living of adults after stroke: a systematic review. Top Stroke Rehabil 2019;26:236-45.

https://doi.org/10.1080/10749357.2019.1574060

27.Kamo T, Momosaki R, Suzuki K, Asahi R, Azami M, Ogihara $\mathrm{H}$, et al. Effectiveness of Intensive Rehabilitation Therapy on Functional Outcomes After Stroke: A Propensity Score Analysis Based on Japan Rehabilitation Database. J Stroke Cerebrovasc Dis 2019;28:2537-42. https://doi.org/10.1016/j.jstrokecerebrovasdis.2019.06.007

28. Malouin F, Richards CL, Durand A, Doyon J. Added value of mental practice combined with a small amount of physical practice on the relearning of rising and sitting post-stroke: a pilot study. J Neurol Phys Ther 2009;33:195-202.

https://doi.org/10.1097/NPT.0b013e3181c2112b

29.Ruffino C, Papaxanthis C, Lebon F. Neural plasticity during motor learning with motor imagery practice: Review and perspectives. Neuroscience 2017;341:61-78.

https://doi.org/10.1016/j.neuroscience.2016.11.023

30.Lanzillo B, Matarazzo G, Calabrese C, Vitale DF. Normalization of Functional Independence Measure variation improves assessment of stroke rehabilitation outcome. Eur J Phys Rehabil Med 2015;51:58796.

https://www.minervamedica.it/en/getfreepdf/tG2kEYRLKEH\%252Btv BalRrQvIBQ0vSBD2CAw6\%252FDxw1CkRosoXqq\%252BRsxmbhQ01j oQ5u\%252BMvfGiymFWBdcvzpYksmMiQ\%253D\%253D/R33Y2015N0 5A0587.pdf 